\title{
Apoyo social percibido: \\ su efecto protector frente a los \\ acontecimientos vitales estresantes
}

\author{
Ana Barrón López de Roda \\ Fernando Chacón Fuertes \\ Universidad Complutense de Madrid
}

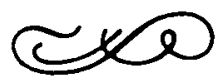

\section{Resumen}

Este estudio examina la relación entre apoyo social percibido, eventos vitales estresantes y depresión en una muestra $(N=74)$ perteneciente a la comunidad de Aranjuez. Como se hipotetizó, aparecen efectos directos significativos del apoyo social y de los eventos vitales sobre la depresión. Sin embargo no se obtuvieron efectos de interacción significativos, no apoyando nuestros resultados la bipótesis del efec. to protector en el caso del apoyo social percibido. Este tipo de apoyo, en vez de simplemente proteger al individuo frente al impacto negativo del estrés, puede ser importante por si mismo en la reducción de la sintomatologia depresiva.

Palabras clave: Apoyo social, estrés.

\section{Perceived social support: its protective effect in stressful events.}

\begin{abstract}
Previous research bas yielded inconsistent, results regarding the mediating effect of social support. Upon psychiatric illness. Using data collectec from a community sample of Aranjuez adults $(N=74)$, the authors examine the relationships among depressive symptoms, perceived social support and stress. ful life events. As anticipated, direct effects were found for perceived support and stressful life events upon depressive symptoms. However, when the data were analyzed via analysis of variance, no statistically significant interactive effects were found. Perceived social support, instead of merely protecting an individual against the negative impact of stress, may itself be important in ameliorating depressive symptomatology.
\end{abstract}

Keywords: Social support, stress.

Dirección de los autores: Departamento de Psicología Social. Campus de Somosaguas, 28023 Madrid.

Original recibido: octubre 1990 
A partir de los años 70, fundamentalmente tras la publicación de los influyentes artículos de Caplan (1974), Cassel $(1974,1976)$ y Cobb (1976), se ha ido aceptando progresivamente el papel del ambiente social tanto como inductor de estrés como reductor del mismo. Es decir, el sistema social es entendido como poseedor de elementos estresores y/o amortiguadores del estrés.

En cuanto a los elementos estresores del ambiente, los investigadores han dirigido su atención hacia los acontecimientos vitales estresantes. Este concepto se ha definido de diferentes formas, haciendo referencia en algunas ocasiones al cambio o reajuste que suponen los eventos en la vida de las personas, y en otros casos apelando a su deseabilidad. Podemos aceptar como definición de los acontecimientos vitales estresantes la siguiente: aquellos eventos que requieren un reajuste en las actividades cotidianas de los individuos y que son percibidos por estos como indeseables (Barrón, 1988).

Respecto a los elementos reductores del estrés se ha prestado especial atención al apoyo social. En este caso se trata de un concepto complejo, que incluye tres niveles de análisis (comunitario, redes sociales e íntimos), que cumple una serie de funciones (emocional, material, informacional), etc.

Lin et al (1986) definen el apoyo social como provisiones instrumentales o expresivas, reales o percibidas, aportadas por la comunidad, redes sociales y amigos íntimos.

La distinción entre real o percibida remite a la polémica respecto a si se debe tener en cuenta sólo los aspectos objetivos del apoyo (apoyo recibido o real) o si hay que tomar en consideración también la dimensión subjetiva del mismo (apoyo percibido). Los autores que subrayan el aspecto subjetivo conceptualizan el apoyo social en términos cognitivos, como percepción de que uno es amado, estimado, etc., por los demás. Se supone que esta percepción promueve la salud (Cobb, 1976).

Tanto el apoyo real como el percibido se relacionan con el bienestar.

Se han hipotetizado dos procesos fundamentales de actuación del apoyo social o dos tipos de efectos (Antonucci y Depner, 1982):

\section{Efectos directos}

Según la hipótesis del efecto directo o principal, a mayor nivel de apoyo social menor malestar psicológico, y a menor grado de apoyo social mayor incidencia de trastornos, independientemente de los acontecimientos vitales estresantes. Esto no quiere decir que en presencia de estresores sociales deje de actuar, sino que el apoyo social ejerce una influencia independiente (Gottlieb, 1983).

\section{Efectos indirectos}

La hipótesis del efecto protector o amortiguador afirma que cuando las personas están expuestas a estresores sociales, estos tendrán efectos negativos sólo entre los sujetos cuyo nivel de apoyo social sea bajo. Sin embargo, según esta hipótesis, sin estresores sociales el apoyo social no tiene influencia sobre el bienestar, su papel se limita a proteger a las personas de los efectos patogénicos del estrés, es esencialmente un moderador de los efectos de los acontecimientos vitales estresantes (Cohen y McKay, 1984; Cohen y Ashby, 1985; Cohen y Syme, 1985).

Diversos estudios empíricos han comprobado la existencia tanto de efectos directos (Berkman y Syme, 1979; Dean y Ensel, 1982; Kaplan et al, 1983) co- 
mo de efectos protectores del apoyo social (Nuckolls et al, 1972; Brown y Bifulco, 1985 Parker y Barnett, 1987).

Ambas hipótesis no son incompatibles, tal y como piensan gran cantidad de investigadores. De hecho, varios estudios hallan ambos tipos de efectos (Gore, 1978; Turner, 1981; Turner, 1981; Aneshensel y Stone, 1982; Cohen y Hoberman, 1983; Kessler y McLeod, 1985).

El presente estudio forma parte de una investigación más amplia, realizada en Aranjuez, sobre los efectos directos y protectores ante el estrés del apoyo social sobre la depresión femenina. En este caso, se presentan los datos referentes al apoyo social percibido.

\section{HIPOTESIS Y DISEÑO}

\section{Hipótesis}

Se hipotetizó que en este aspecto el apoyo social tendría tanto efectos directos sobre la depresión como efectos protectores frente a los acontecimientos vitales estresantes.

\section{Diseño}

Se trata de un estudio comparativo, cuasi-experimental, ya que se trata de variables de selección, con un diseño factorial $2 \times 2$ con medidas independientes y grupos asignados.

La variable dependiente era la depresión y como posibles variables independientes se utilizaron el estrés psicosocial y el apoyo social percibido.

\section{METODO}

\section{Instrumentos}

a) Life Experiences Survey (Sarason et al., 1978).

Este cuestionario fue seleccionado como medida de los acontecimientos vitales estresantes. Proporciona una medida indirecta del estrés vital que surge ante los eventos ocurridos durante el último año de la vida del individuo. Entre los cuestionarios existentes se escogió éste porque incluye medidas de dos aspectos fundamentales de los eventos vitales: su deseabilidad e impacto subjetivo.

Divide el período de un año en subperíodos de seis meses, permitiendo estudiar si el efecto negativo de los acontecimientos vitales estresantes se debe a los eventos ocurridos inmediatamente antes del desarrollo del trastorno, o si es mejor considerar el año completo.

Este cuestionario ha sido traducido y adaptado a la población española para estas investigaciones, y se le ha añadido una nueva dimensión; predictibilidad o esperabilidad de los eventos. Se consideró oportuna su inclusión, tal y como hicieron Sarason et al (1983), ya que este factor aparece frecuentemente en la literatura moderando la relación entre estrés psicosocial y salud. Por tanto, además de las ya clásicas puntuaciones de acontecimientos indeseables o negativos, se obtuvieron puntuaciones corregidas teniendo en cuenta la esperabilidad de cada evento.

Los sujetos sólo deben contestar a los ítems que hayan experimentado. En este caso, deben responder: 
- Mes de ocurrencia.

-Impacto percibido que tuvo en sus vidas. Este aspecto se contestaba mediante una escala de 7 puntos, de $+3 a-3(+3$ significa muy positivo y -3 muy negativo).

-Esperabilidad del evento, desde totalmente esperable (a lo que correspondía una puntuación de 1), hasta nada esperable (con una puntuación de 4 en este caso).

Los datos sobre su fiabilidad aparecen en Barrón (1988).

b) Cuestionario de Recursos Sociales (Díaz-Veiga, 1987).

Este cuestionario se seleccionó como medida del apoyo social y fue originalmente elaborado para ancianos. Para esta investigación ha sido adaptado a la población objeto de estudio, modificando un factor. Se sustituyó la interacción con los hijos por la interacción con los vecinos. Se consideró oportuno este cambio dadas las características de la población objeto de estudio (una comunidad pequeña) y aparecer frecuentemente en la literatura relativa al tema.

Proporciona medidas de distintos aspectos del apoyo social: áreas de interacción del sujeto, tipo de apoyo recibido en cada área, y grado de satisfacción con el mismo. Los datos presentados se refieren a este último aspecto. Los datos sobre su fiabilidad se encuentran en Díaz Veiga (1987).

c) La variable de salud seleccionada fue la depresión, y como medida de la misma se utilizó el cuestionario de Zung (1965). Los datos de fiabilidad de la adaptación española aparecen en Barrón (1988).

\section{Muestra}

Consta de 74 mujeres pertenecientes a la comunidad de Aranjuez, todas ellas de nivel socioeconómico bajo y medio-bajo, y de edades comprendidas entre los 25 y 65 años.

\section{Procedimiento}

A todos los sujetos se les aplicaron los instrumentos descritos anteriormente. Los cuestionarios fueron aplicados en forma de entrevista dado el bajo nivel educativo de las mujeres que formaban la muestra. Asimismo, al final de la entrevista se les preguntaba si habían experimentado algún otro evento que hubiera tenido un impacto importante en sus vidas y que no se les hubiera nombrado. Se trataba de solucionar el problema de que la muestra de eventos no fuese completa.

En concreto las puntuaciones que se obtuvieron fueron:

1. Puntuación de acontecimientos vitales negativos de todo el año. Es la suma de las puntuaciones de impacto percibido de aquellos eventos experimentados y evaluados negativamente del año completo.

2. Puntuación corregida con esperabilidad. La puntuación otorgada por el sujeto a cada evento se multiplicó por su puntuación correspondiente en esperabilidad. Posteriormente, se sumaron y se obtuvo una puntuación similar a la anterior pero teniendo en cuenta la predictibilidad.

3. Puntuaciones de subperíodos de seis meses. Se obtuvieron puntuaciones equivalentes a las dos descritas anteriormente para cada subperíodo de seis meses, es decir, acontecimientos negativos de los seis meses inmedia- 
tamente anteriores al inicio del trastorno y del primer subperíodo de seis meses, y puntuaciones corregidas con esperabilidad de ambos subperíodos.

4. Apoyo social percibido. Grado de satisfacción con el apoyo recibido de todas las áreas de interacción del sujeto. Se sumaron las puntuaciones de satisfacción de cada área de interacción, las cuales oscilaban entre 1 (poco satisfecho) y 3 (muy satisfecho).

Una vez aplicados los cuestionarios los sujetos fueron clasificados según la mediana de las puntuaciones en cada uno de los cuestionarios.

A continuación se pasó al análisis de datos.

\section{Análisis de datos}

Se aplicó un análisis de varianza mediante el paquete de programas BMDP (BMDP2V). El análisis de varianza nos permitiría estudiar tanto los efectos directos de las variables sobre la depresión, como sus efectos de interacción.

En primer lugar se realizaron los análisis correspondientes a las puntuaciones de estrés del año completo, y posteriormente las de los subperíodos de seis meses.

Como es bien sabido, para poder aplicar el análisis de varianza paramétrico es preciso comprobar el principio de homocedasticidad, o igualdad de varianzas entre los grupos. Por ello, previamente a cada análisis se aplicó la prueba de Barlett.

\section{RESULTADOS Y CONCLUSIONES}

\section{Resultados}

Los análisis mostraron efectos directos sobre la depresión de las siguientes variables:

1. Eventos negativos del año completo $(F=25.18, p \leq 0.00)$.

2. Eventos negativos del año completo teniendo en cuenta la esperabilidad $(\mathrm{F}=27.06, \mathrm{p} \leq 0.00)$.

3. Eventos negativos ocurridos en los seis meses inmediatamente anteriores al inicio del trastorno $(F=10,66, p \leq 0.00)$.

4. Eventos negativos de los seis meses inmediatamente anteriores al inicio del trastorno teniendo en cuenta la esperabilidad de los mismos ( $F=11,83$, $\mathrm{p} \leq 0.00)$.

5. Apoyo social percibido $(\mathrm{F}=5,93, \mathrm{p} \leq 0.01)$.

No aparecen efectos directos de las puntuaciones de estrés del primer subperíodo del año. Asimismo, en ningún caso aparecen efectos de interacción entre el apoyo social percibido y ninguna de las puntuaciones de estrés vital.

\section{Conclusiones}

Los análisis de varianza muestran que los açontecimientos vitales estresantes tienen un efecto directo significativo sobre là depresión. Además, este efecto parece deberse a los eventos ocurridos en los seis meses inmediatamente anteriores al comienzo del trastorno. Este hallazgo es consistente con investigaciones anteriores (Barrett, 1979; Paykel, 1985; Katschnig, 1986). 
Respecto al apoyo social percibido, estos resultados son consistentes con la literatura que existe a favor de los efectos directos del apoyo social sobre la salud (Schaefer et al, 1981; Williams et al, 1981, Bell et al, 1982). Sin embargo, no se confirma la hipótesis del efecto protector frente al estrés, ya que en ningún caso aparecen efectos de interacción entre ambas variables.

En cualquier caso, no se debe concluir definitivamente que no existen este tipo de efectos. Es posible que otros aspectos del apoyo social sí muestren estos efectos de amortiguación.

Se deberían investigar aspectos funcionales y estructurales del apoyo y su posible interacción con los acontecimientos vitales estresantes.

En cualquier caso, se ha mostrado cómo el ambiente social influye en el bienestar y en la salud de las personas, lo que debería dirigirse en el futuro a desarrollo de programas de prevención, tanto a nivel primario como secundario.

\section{Referencias}

Aneshensel, C.S. y Stone, J.D. Stress and depression: A test of the buffering model of social support. Archives of General Psychiatry, 1982, 39, 1392-1396.

Antonucci, T.C. y Depner, C.R. Social support and informal helping relationships. En: T.A. Wills. Basic processes in belping realtionships. Academic Press, New York, 1982.

Barrett, J.E. Stress and mental disorder. Raven Press, New York, 1979.

Barron, A. Estrés psicosocial, apoyo social y depresión en mujeres. Un estudio empírico en Aranjuez. Tesis Doctoral. Facultad de Psicología. U.C.M. 1988.

BezL, R.A.; Leroy, J.B.; y STEPHENSON, J.J. Evaluating the mediating effects of social support upon life events and depression symptoms. Joumal of Community Psychology, 1982, $10,4,325-340$.

Brekman, L.F. y Syme, S.L. Social networks, host resistance and mortality: A nine year follow-up study of Alameda Country residents. American Joumal of Epidemiology, 1979, 109, 186-204.

Brown, G.W. y Bifulco, A. Social support, life events and depression. En: I.G. Sarason; y B.R. Sarason. Social support: Theory, research and applications. Martinus Nijhoff, Boston, 1985.

CaPLAN, G. Social support and community mental bealth. Basic Books, New York, 1974.

CASSEL, J. Psychosocial processes and stress: Theoretical formulations. International Journal of Human Services, 1974, 4, 3, 471-482.

Cassel, J. The contribution of social environment to host resistance. American Joumal of Epidemiology, 1976, 104, 2, 107-123.

Соов, S. Social support as a moderator of life stress. Psychosomatic Medicine, 1976, 38, 5 , 300-314.

Conen, S. y Hoberman, H. Positive events and social support as buffers of life changes stress. Joumal of Applied Social Psychology, 1983, 13, 2, 99-125.

Cohen, S; y McKay, G. Social support, stress and the buffering hypothesis: A theoretical analysis. En: A. Baum; S.E. Taylor; y J.E. Singer. Handbook of Psychology and health. Hillsdale, New Jersey, 1984.

Conen, S y AshBy, T. Stress, social support and the buffering hypothesis. Psychological Bulletin, 1985, 98, 2, 310.357.

Cohen, S y Syme, L. Social support and bealth. Academic Press, New York, 1985.

DEAN, A y ENSEL, W.M. Modeling social support, life events, competence, and depression in the context of age an sex. Joumal of Community Psychology, 1982, 10, 392-408.

Díazveiga, P. Evaluación del apoyo social. En: R. Fernández Ballesteros. El ambiente: Análisis psicológico. Pirámide, Madrid, 1987.

Gore, S. The effects of social support in moderating the health consequences of unemployement. Journal of Health and Social Behavior, 1978, 19, 157-165. 
Gotture, B.H. Social support strategies. Sage, Beverly Hills, 1983.

Kaplan, H.B.; RobBins, C. y Martin, S.S. Antecedents of psychological distress in young adults: self rejection, deprivation of social support and life events. Joumal of Health and Social Behavior, 1983, 24, 3, 230-244.

KatSCHNIG, H. Life events and psychiatric disorders: Controversial issues. Cambridge University Press, Cambridge, 1986.

KESSLER, R.C. Y McLEOD, J. Social support and mental health in community samples. En: S. Cohen; y L. Syme. Social support and bealth. Academic Press, New York, 1985.

Lin, N; Dean, A. y Ensel, W.M. Social support, life events and depression. Academic Press, New York, 1986.

Nuckolls, K.B.; Cassel, J; y Kaplan, B.H. Psychosocial assets, life crisis and the prognosis of pregnancy. American Joumal of Epidemiology, 1972, 95, 431-441.

Parker, G. y BarnetT, B. A test of the social support hypothesis. British Joumal of Psychiatry, 1987, 150, 72-77.

PAYKel, E.S. Life events, social support and clinical psychiatric disorer En: I.G. Sarason; y B.R. Sarason. Social support: Theory, esearch and applications. Martinus Mijhoff, Boston, 1985.

Sarason, I.G.; Johnson, J.H. y Siegel, J.M.. Assesing the impact of life changes: Development of the Life Experiencies Survey. En: T.G. Sarason; y C.D. PILBERGER. Stress and anxiety. Halsted Press, New York, 1978.

Sarason, I.G.; Levine, H.M; Basham, R.B. y Sarason, B.R. Assesing social support: The social support questionnaire. Joumal of Pesonality and Social Psychology, 1983, 44, 1, 127-139.

SCHAEFER, C; Coyne, J.C. y Lazarus, R.S. The health related functions of social support. Journal of Bebavioral Medicine, 1981, 4, 4, 381-406.

TURNER, R.J. Direct, indirect and moderating effects of social support on psychological distress and associates conditions. En: H.B. Kaplan. Pychosocial stress: Trends in theory and. research. Academic Press, New York, 1983.

W Illiams, A.W.; Ware, J.F.E. y Donald, C.A. A model of mental halth, life events and social supports applicable to general populations. Joumal of Health and Social Behavior, 1981, $22,4,324-336$.

Zung, W.W.K. A self rating depression scale. Arcbives of General Psycbiatry, 1965, 12, 63-70. 